\title{
Fotossíntese e relações hídricas de duas cultivares jovens de seringueira
}

\author{
JULIANE RIBEIRO CAVALCANTE ${ }^{1,3}$ e ELENICE DE CÁSSIA CONFORTO²
}

(recebido: 5 de maio de 2005; aceito: 23 de novembro de 2006)

\begin{abstract}
Photosynthesis and water relations of two young rubber tree cultivars). This work aimed to compare some aspects of the leaf-gas exchange and water balance of two field grown cultivars (Fx 3864 e RRIM 600) of the rubber tree. Measurements were carried out for two months between the wet and dry seasons of 2001 and 2002. Integrated values of gas exchange showed a decrease in photosynthesis (32\% and 22\%), transpiration (44\% and 38\%), and stomatal conductance (29\% and 17\%) for RRIM 600 and Fx 3864, respectively. Osmotic potential decreased by 41\% for RRIM 600 , and by $36 \%$ for Fx 3864 . The results suggest that the performance of Fx 3864 was similar to that of RRIM 600 throughout the year at the juvenile stage studied.
\end{abstract}

Key words - gas exchange, Hevea brasiliensis, water and osmotic potentials

RESUMO - (Fotossíntese e relações hídricas de duas cultivares jovens de seringueira). Este trabalho teve como objetivo comparar aspectos das trocas gasosas e das relações hídricas foliares da cultivar Fx 3864 e da cultivar RRIM 600, já estabelecida em larga escala no pólo produtor de borracha de São José do Rio Preto, SP. As cultivares foram mantidas em condições de campo, onde foram realizadas medidas das trocas gasosas e do potencial osmótico e hídrico, durante dois meses, dentro dos períodos chuvoso e seco, nos anos de 2001 e 2002. Valores integralizados das trocas gasosas foliares mostraram que, entre os períodos chuvoso e seco, ocorreu um decréscimo da taxa fotossintética de $32 \%$ e $22 \%$, da transpiração de $44 \%$ e $38 \%$ e da condutância estomática de 29\% e 17\% para RRIM 600 e Fx 3864, respectivamente. Durante o período seco os valores médios de potencial osmótico decresceram 41\% para RRIM 600, e 36\% para Fx 3864. Os resultados sugerem que o desempenho da cultivar Fx 3864 foi equivalente à de RRIM 600 durante a fase jovem estudada.

Palavras-chave - Hevea brasiliensis, trocas gasosas, potenciais hídrico e osmótico

\section{Introdução}

O Estado de São Paulo tem liderado a produção brasileira de borracha natural, com cerca de 45.000 hectares plantados com seringueira, abrangendo mais de 2.500 produtores (Cortez \& Benesi 2000). No pólo de produção em torno de São José do Rio Preto, as condições edafoclimáticas favorecem a heveicultura por apresentar menor freqüência de geadas e ventos frios, melhor estabilidade térmica (Instituto Agronômico de Campinas 2002), bem como solos adequados (Bataglia et al. 1987). Os seringais paulistas apresentam produtividade média entre 1.200 e $1.500 \mathrm{~kg} \mathrm{ha}^{-1} \mathrm{ano}^{-1}$, acima da média dos tradicionais países produtores, como Tailândia, Indonésia e Malásia, cujas produtividades médias variam de 750 a $1.100 \mathrm{~kg} \mathrm{ha}^{-1} \mathrm{ano}^{-1}$, conferindo

\footnotetext{
1. Universidade Federal de São Carlos, Programa de PósGraduação em Ecologia e Recursos Naturais, Rodovia Washington Luís km 235, Monjolinho, Caixa Postal 676, 13565-905 São Carlos, SP, Brasil.

2. Universidade Estadual Paulista, Instituto de Biociências, Letras e Ciências Exatas, Departamento de Zoologia e Botânica, Rua Cristóvão Colombo, 2265, Jardim Nazareth, 1554-000 São José do Rio Preto, SP, Brasil.

3. Autor para correspondência: jrc_biologa@yahoo.com.br
}

ao Estado de São Paulo, portanto, destaque dentre as regiões mais produtivas do mundo (Instituto Agronômico de Campinas 2002).

A produtividade da seringueira é controlada por fatores genéticos, ecológicos e fisiológicos. Devido ao descompasso entre a produção e a demanda de borracha natural, um maior conhecimento sobre o comportamento das plantas nas diferentes condições de plantio são importantes, pois uma cultivar melhor adaptada reduz 0 período improdutivo do seringal gerando maior retorno econômico ao produtor. Nesse sentido, novas cultivares de seringueira adaptadas a diferentes regiões ecológicas são importantes para o sucesso da heveicultura (Gonçalves 1998).

A cultivar RRIM 600 é plantada em larga escala (70\% da área total de plantio) no Planalto Ocidental Paulista, pelo seu destaque na produção (Gonçalves 1998); a cultivar Fx 3864 é plantada em larga escala na região de Mato Grosso. No Estado de São Paulo esta cultivar é plantada em escala experimental ( $28 \%$ da área total de plantio), nos municípios de Buritama e Presidente Prudente. A expansão do plantio da cultivar Fx 3864 sofre resistência pois, até o momento, não há estudos sobre seu desenvolvimento vegetativo, nem dados ecofisiológicos que permitam comparar suas respostas 
às condições climáticas do Planalto Ocidental Paulista, especificamente na fase jovem.

O substrato para a síntese de látex depende, em curto prazo, da fotossíntese. Portanto, determinações da taxa fotossintética ao longo do ano podem fornecer informações valiosas sobre flutuações na quantidade do látex. Há uma estreita relação entre a turgescência e o fluxo de látex. Nesse sentido, o potencial osmótico e hídrico foliar refletem o comportamento fisiológico da seringueira, principalmente no período seco (Brunini \& Cardoso 1998). Alguns trabalhos sobre a fotossíntese e as relações hídricas em seringueira têm sido realizados com sucesso em condições de laboratório (Rocha Neto et al. 1983, Conceição et al. 1985, Cascardo et al. 1993, Brunini \& Cardoso 1998); contudo, são raros os trabalhos que investigam tais aspectos em condições de campo, onde a variação simultânea dos fatores ambientais torna o estudo mais complexo, mas é fundamental para a compreensão dos processos adaptativos das espécies agrícolas (Machado \& Lagôa 1994).

O presente estudo objetivou comparar as relações hídricas e trocas gasosas da cultivar Fx 3864 em relação à RRIM 600, em condições de campo, nos períodos chuvoso e seco; e inferir sobre a possibilidade do uso de cultivar Fx 3864 como alternativa para plantio comercial em São José do Rio Preto, SP, Planalto Ocidental Paulista.

\section{Material e métodos}

Utilizaram-se plantas jovens de seringueira [Hevea brasiliensis (Willd. ex A. Juss.) Müll. Arg.], Euphorbiaceae, cultivar RRIM 600, proveniente da Fazenda São João (Olímpia, SP), e cultivar Fx 3864, proveniente de Nhandeara, SP, ambas enxertadas sobre a cultivar $\mathrm{Tj} 16 . \mathrm{O}$ experimento foi instalado em outubro/2000 em condições de campo, na área experimental do Departamento de Zoologia e Botânica da Universidade Estadual Paulista, no Instituto de Biociências, Letras e Ciências Exatas (UNESP/IBILCE), campus de São José do Rio Preto (2049'11' S e 49 22'46” W), norte do Estado de São Paulo. O solo da área experimental é do tipo argissolo vermelho-amarelo, com textura arenosa média, em fase de relevo suave ondulado (Empresa Brasileira de Pesquisa Agropecuária 1999). As cultivares foram dispostos em duas parcelas distantes 2,0 m entre si. Cada parcela foi constituída por cinco fileiras, contendo 10 plantas cada, sob espaçamento de $1,0 \mathrm{~m} \times 0,9 \mathrm{~m}$. As amostragens e medições foram feitas durante os anos de 2001 e 2002, sendo escolhidos os meses mais chuvosos (dezembro/2001 e fevereiro/2002) e os mais secos (julho e agosto/2002 - estiagem de inverno), para análise neste trabalho. Nestes meses, as plantas estavam na fase juvenil.
As trocas gasosas e os parâmetros microclimáticos durante o curso do dia foram determinados com um analisador de gás no infravermelho (LCA-4, ADC, Hoddesdon, Reino Unido), entre $8 \mathrm{~h} 00$ e $15 \mathrm{~h} 30$, por compreender o período mais favorável (manhã) e menos favorável (logo após o meio dia) para as trocas gasosas (Prado \& Moraes 1997, Eamus et al. 1999). As medidas foram realizadas em dias claros e sem nuvens, em intervalos regulares de 60 minutos, em três plantas de cada cultivar, com cinco determinações por planta. Utilizaram-se folhas intactas não destacadas, totalmente expandidas, sob condições naturais de luz e $\mathrm{CO}_{2}$, sem sinais de herbivoria, doenças ou senescência. A cada determinação, esperava-se cerca de um minuto para atingir-se novamente o equilíbrio da leitura. Em julho e agosto de 2002, foi necessário o uso de andaimes para atingir as folhas úteis. Assumindose que não há variação na resistência estomática entre os folíolos laterais e medianos (Conforto et al. 1998), a câmara de inserção do limbo foliar (PLCB-4, ADC) foi posicionada no folíolo mediano, evitando-se as nervuras e o bordo foliar. Durante o curso do dia foram determinados os valores médios e os integralizados (Prado et al. 2001) da taxa fotossintética líquida (A), transpiração (E), condutância estomática $\left(\mathrm{g}_{\mathrm{s}}\right)$, temperatura da folha $\left(\mathrm{T}_{\mathrm{f}}\right)$, e parâmetros microclimáticos como a radiação fotossinteticamente ativa (RFA). Os valores da eficiência do uso da água $\left(\mathrm{E}_{\mathrm{A}}\right)$ foram obtidos por meio da razão A/E (Kramer \& Boyer 1995).

As curvas de resposta da fotossíntese líquida a radiação fotossinteticamente ativa (A-RFA) também foram obtidas com o analisador de gás no infravermelho citado, entre $9 \mathrm{~h} 00 \mathrm{e}$ $10 \mathrm{~h} 00$, em dias e folhas com as mesmas características daquelas utilizadas para as trocas gasosas. Foram realizadas duas curvas, em duas plantas de cada cultivar, nos períodos chuvoso e seco. Como os valores obtidos nos períodos chuvoso e seco foram estatisticamente semelhantes em ambas cultivares, utilizou-se para discussão apenas uma curva de cada cultivar. As medidas foram feitas em condições decrescentes de RFA, começando em radiação plena, cerca de $1.500 \mu \mathrm{mol} \mathrm{m}{ }^{-2} \mathrm{~s}^{-1}$, até atingir-se intensidade zero. A atenuação da luz incidente foi obtida mediante a sobreposição de telas negras de plástico tipo sombrite, simultaneamente sobre a câmara foliar e sobre o sensor de RFA. A cada troca de tela, esperava-se cerca de 20 s para atingir-se novamente o equilíbrio (Long \& Hallgren 1985). Para o ajuste da curva e obtenção dos valores da taxa fotossintética (A), irradiância de saturação $\left(I_{s}\right)$ e da irradiância da compensação $\left(I_{c}\right)$, foi utilizada a equação proposta por Prado \& Moraes (1997). Com a primeira derivada desta equação foi calculada a eficiência quântica aparente (Ø).

O potencial osmótico foliar $(\psi \pi)$ foi determinado com higrômetro (HR 33T, Wescor, EUA) calibrado no ponto de orvalho (Neumann \& Thurtell 1972), nos horários correspondentes às medidas das trocas gasosas, em três plantas de cada cultivar, com cinco determinações por planta. O potencial hídrico foliar $(\psi)$ foi obtido com câmara de pressão (3005, Santa Barbara Soil Moisture, EUA) (Scholander et al. 
1965), nos horários correspondentes às medidas de trocas gasosas, em três plantas de cada cultivar, com cinco determinações por planta, em julho/2002 (período seco). A medida de foi efetuada após a formação de um coágulo de látex (Cascardo et al. 1993).

Os valores diários de chuva e da umidade relativa do ar durante o período de estudo foram obtidos junto à Secretaria de Agricultura e Abastecimento (Núcleo de Produção de Sementes, São José do Rio Preto). Para o cálculo dos valores médios das trocas gasosas, $\psi \pi, \psi$, ajuste da curva A-RFA e integralização dos dados das trocas gasosas (Prado et al. 2001), foi utilizado o software Microcal Origin (Microcal Origin 4.0 1995). Os dados integralizados foram submetidos ao Teste $\mathrm{F}$ (Zar 1999).

\section{Resultados e Discussão}

Parâmetros ambientais - Os valores médios da precipitação mensal, das temperaturas máxima, mínima e da umidade relativa do ar durante os anos de $2001 \mathrm{e}$ 2002 são apresentados na figura 1. Foram escolhidos para discussão dos experimentos, dois períodos contrastantes: o chuvoso (dezembro/2001 e

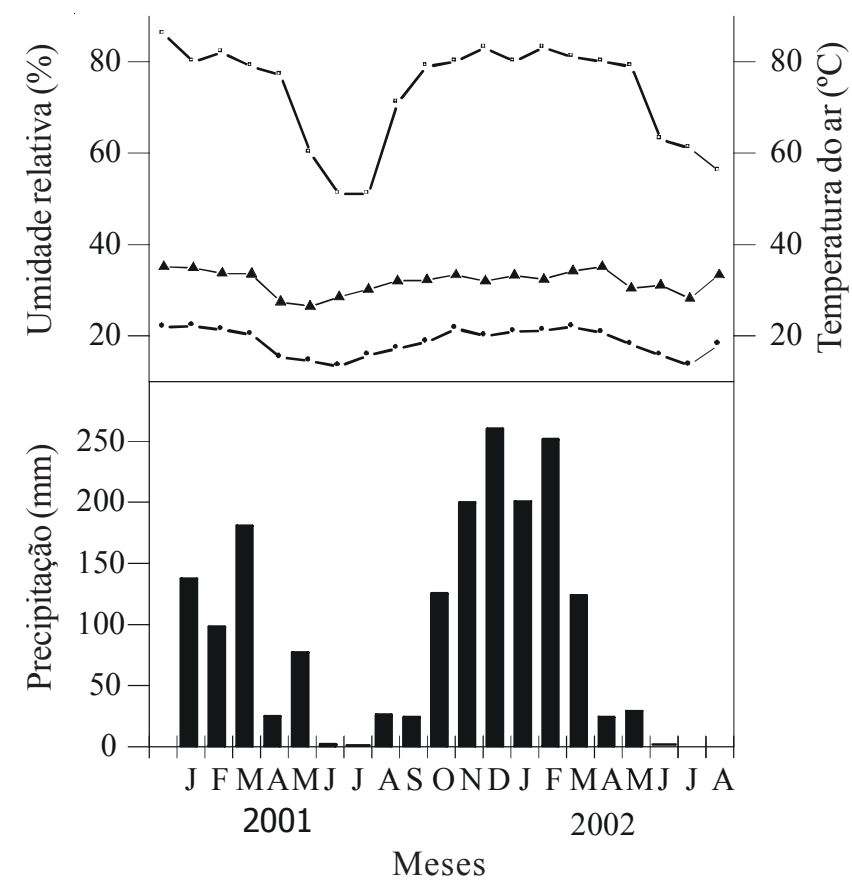

Figura 1. Valores médios da umidade relativa $(\square)$ e temperaturas máxima $(\boldsymbol{\Delta})$ e mínima $(\bullet)$ do ar e precipitação mensal durante os anos de 2001 e 2002 em São José do Rio Preto, SP.

Figure 1. Mean values of air relative humidity $(\square)$, maximum $(\Delta)$ and minimum $(\bullet)$ air temperature and total monthly rainfall during the years of 2001 and 2002 in Sao Jose do Rio Preto, SP. fevereiro/2002) e o seco (julho e agosto/2002). As maiores precipitações no período chuvoso estiveram acima de $250 \mathrm{~mm}$, e as menores no período seco, abaixo de $25 \mathrm{~mm}$.

Trocas gasosas - Os cursos diários são apresentados na figura 2. Os valores mostraram, para as duas cultivares, diferenças entre os períodos seco e chuvoso. Contudo, a oscilação diária não seguiu, para todos os parâmetros, um comportamento definido, tendo havido decréscimos substanciais em $\mathrm{g}_{\mathrm{s}}$ não acompanhados pelo equivalente decréscimo na E. Este padrão de comportamento é relatado na literatura (Dai et al. 1992). De fato, sob condições de campo, a variação simultânea dos fatores ambientais torna o estudo mais complexo, e a análise pontual das trocas gasosas durante o dia pode fornecer conclusões equivocadas. Neste sentido, a integralização dos valores das trocas gasosas é mais representativa do desempenho da cultivar durante o curso do dia em diferentes épocas do ano (Prado et al. 2001, Conforto et al. 2005).

A integralização dos valores (tabela 1) revelou que as diferenças entre as cultivares no período chuvoso não foram estatisticamente significativas e no período seco, com exceção de A, os demais valores diferiram significativamente. Houve decréscimo da taxa fotossintética de $32 \%$ e $22 \%$, da transpiração de $44 \%$ e $38 \%$ e da condutância estomática de $29 \%$ e $17 \%$ para RRIM 600 e Fx 3864, respectivamente. Provavelmente, a diminuição nesses valores deveu-se a menor disponibilidade de água no solo ou ao maior déficit de pressão de vapor do ar no período seco. $\mathrm{O}$ decréscimo dos valores de $\mathrm{A}$ e $\mathrm{E}$ foram equivalentes aos observados por Cavalcante \& Conforto (2002) em plantas jovens de seringueira, no período seco, em São José do Rio Preto (decréscimo de $34 \%$ de A e $44 \%$ de E).

$\mathrm{O}$ padrão de comportamento da $\mathrm{A}$ foi equivalente entre as cultivares durante todo o período de estudo. A redução nos valores de $\mathrm{g}_{\mathrm{s}}$ e de $\mathrm{E}$, observada para ambas as cultivares, sugeriu comportamento preventivo à desidratação do tecido, evitando dessecação maior no período seco, aumentando, conseqüentemente, a $\mathrm{E}_{\mathrm{A}}$ (Turner 1986, Brunini \& Cardoso 1998).

Potencial hídrico e osmótico - A determinação do $\psi$ foi realizada após 35 dias sem chuva. Os menores valores observados foram de -1,80 MPa para RRIM 600, e de -1,70 MPa para Fx 3864 (figura 3); nestas condições, os estômatos não se fecharam totalmente. Em plantas jovens de seringueira, envasadas, Cascardo et al. (1993) relataram, o fechamento total dos estômatos, após 13 dias sem rega, sob $\psi$ de $-1,3 \mathrm{MPa}$. Porém, sob condições de campo, o déficit hídrico desenvolve-se gradualmente. 
No período seco, houve diminuição média no $\psi \pi$, de $41 \%$ para RRIM 600 , e de $36 \%$ para Fx 3864 , possivelmente associada às reduções de $\mathrm{E} e \mathrm{~g}_{\mathrm{s}}$ verificadas. Os níveis de redução observados para o $\psi \pi$, permitem inferir que houve ajustamento osmótico à medida que o estresse hídrico foi se acentuando, possibilitando a aclimatação e, conseqüentemente, a manutenção, pela manhã, de valores de $\psi$ próximos aos de plantas hidratadas (entre $-0,5 \mathrm{MPa}$ e $-1,0 \mathrm{MPa}$, segundo Brunini \& Cardoso 1998). Se os valores de $\psi \pi$ do período chuvoso fossem mantidos no período seco, a pressão de parede seria nula durante todo o curso do dia, em julho ou em agosto, para ambas as cultivares estudadas.

A manutenção da abertura estomática em períodos com baixa disponibilidade hídrica é verificada em algumas plantas e deve-se a sua capacidade de extrair água do solo rápido o suficiente para compensar as
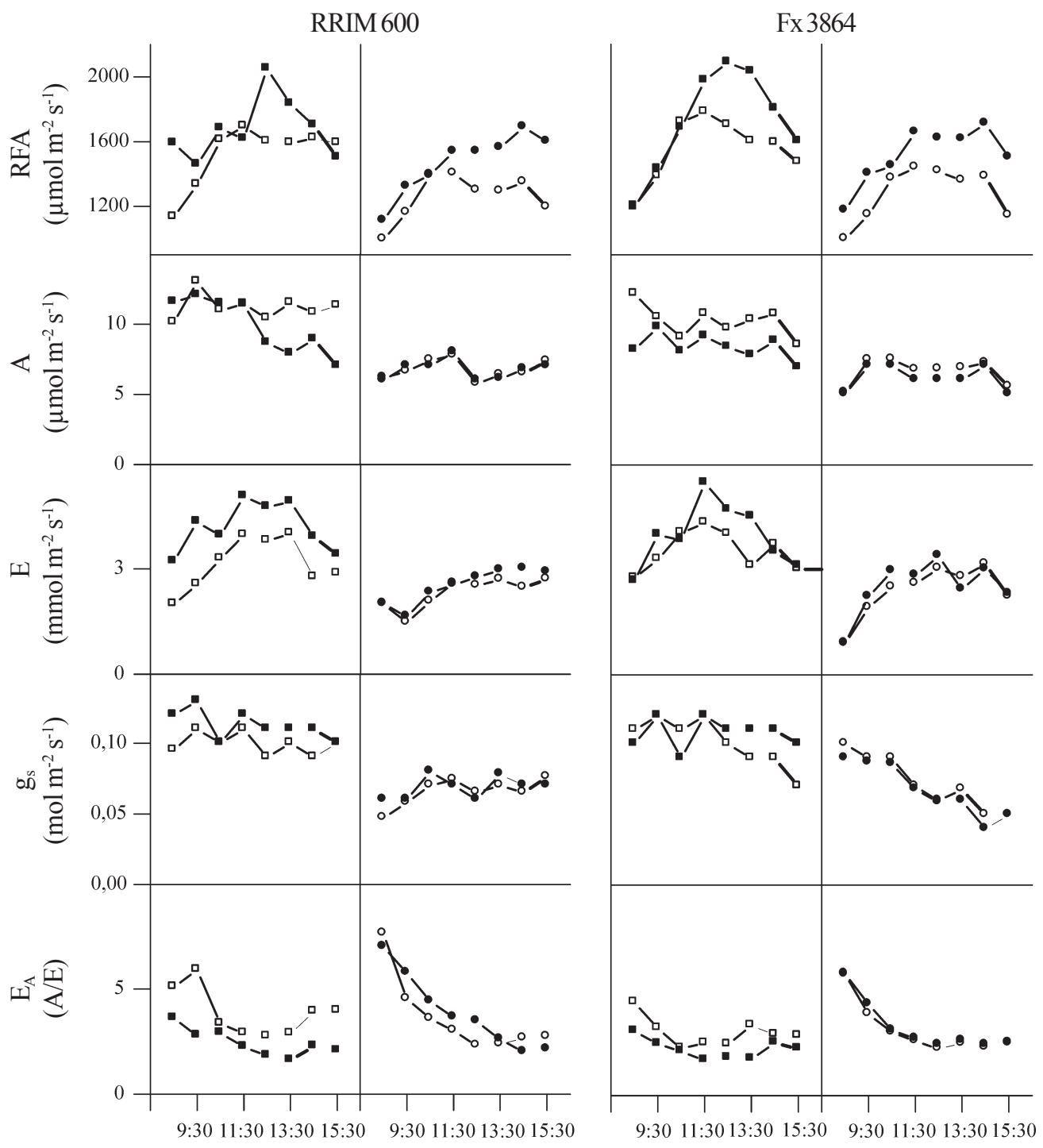

Hora do dia

Figura 2. Variações na radiação fotossinteticamente ativa (RFA), taxa fotossintética líquida (A), transpiração (E), condutância estomática $\left(\mathrm{g}_{\mathrm{s}}\right)$ e eficiência do uso da água ( $\mathrm{E}_{\mathrm{A}}$ ), obtidas nas cultivares RRIM 600 e Fx 3864 de Hevea brasiliensis (Willd. ex A.

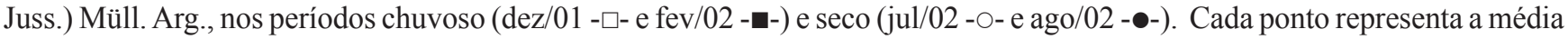
de quinze determinações.

Figure 2. Variations in the photosynthetic active radiation (PAR), net photosynthesis rate (A), transpiration (E), stomatal conductance $\left(\mathrm{g}_{\mathrm{s}}\right)$ and water use efficiency $\left(\mathrm{E}_{\mathrm{A}}\right.$ ), of cultivars RRIM 600 and Fx 3864 of the H. brasiliensis (Willd. ex A. Juss.)

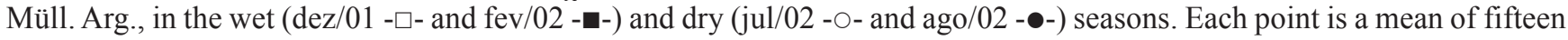
determinations. 
Tabela 1. Valores integralizados da temperatura média da folha $\left(\mathrm{T}_{\mathrm{f}},{ }^{\circ} \mathrm{C}\right)$, fotossíntese líquida $\left(\mathrm{A}, \mathrm{mmol} \mathrm{m}^{-2}\right.$ dia $\left.^{-1}\right)$, transpiração $\left(\mathrm{E}, \mathrm{mol} \mathrm{m}^{-2} \mathrm{dia}^{-1}\right)$, condutância estomática $\left(\mathrm{g}_{\mathrm{s}}, \mathrm{mol} \mathrm{m}^{-2} \mathrm{dia}^{-1}\right)$ e eficiência do uso da água $\left(\mathrm{E}_{\mathrm{A}}, \mathrm{A} / \mathrm{E}\right)$, nos períodos chuvoso e seco, nas cultivares, RRIM 600 e Fx 3864, de Hevea brasiliensis (Willd. ex A. Juss.) Müll. Arg., sob condições de campo.

Table 1. Integrated values of the leaf mean temperature $\left(\mathrm{T}_{\mathrm{f}},{ }^{\circ} \mathrm{C}\right)$, net photosynthesis $\left(\mathrm{A}, \mathrm{mmol} \mathrm{m}^{-2}\right.$ day $\left.^{-1}\right)$, transpiration $\left(\mathrm{E}, \mathrm{mol} \mathrm{m} \mathrm{m}^{-2}\right.$ day $\left.^{-1}\right)$, stomatal conductance $\left(\mathrm{g}_{\mathrm{s}}, \mathrm{mol} \mathrm{m}^{-2}\right.$ day $\left.^{-1}\right)$, and water use efficiency $\left(\mathrm{E}_{\mathrm{A}}, \mathrm{A} / \mathrm{E}\right)$, in the wet and dry seasons, of cultivars, RRIM 600 and Fx 3864, of the H. brasiliensis (Willd. ex A. Juss.) Müll. Arg., under field conditions.

\begin{tabular}{|c|c|c|c|c|c|c|c|c|c|c|c|}
\hline \multirow[t]{2}{*}{ Parâmetros } & \multicolumn{5}{|c|}{ RRIM 600} & \multicolumn{3}{|c|}{ Fx 3864} & \multirow{2}{*}{$\begin{array}{l}\text { Período } \\
\text { chuvoso } \\
-\end{array}$} & \multirow{2}{*}{$\begin{array}{c}\text { Período } \\
\text { seco }\end{array}$} & \multirow{2}{*}{ 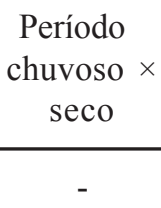 } \\
\hline & $\operatorname{dez} / 01$ & $\mathrm{fev} / 02$ & jul/02 & ago/02 & $\mathrm{dez} / 01$ & $\mathrm{fev} / 02$ & jul/02 & ago/02 & & & \\
\hline $\mathrm{T}_{\mathrm{f}}$ & 36,00 & 35,00 & 26,00 & 24,00 & 35,00 & 37,00 & 28,00 & 23,00 & - & - & - \\
\hline$A^{1}$ & 425,82 & 376,11 & 65,46 & 261,63 & 384,97 & 321,19 & 269,05 & 275,67 & $2,80 \mathrm{~ns}$ & $10,62 \mathrm{~ns}$ & $21,15^{* *}$ \\
\hline $\mathrm{E}$ & 123,04 & 163,27 & 82,35 & 77,62 & 136,70 & 155,76 & 90,15 & 89,48 & $0,03 \mathrm{~ns}$ & $33,87 * *$ & $19,30 * *$ \\
\hline $\mathrm{g}_{\mathrm{s}}$ & 3766,50 & 4266,00 & 2800,00 & 2889,00 & 3936,60 & 4104,00 & 3429,00 & 3213,00 & $0,0047 \mathrm{~ns}$ & $33,28 * *$ & $31,75^{* *}$ \\
\hline $\mathrm{E}_{\mathrm{A}}$ & 3,46 & 2,30 & 3,20 & 3,37 & 2,82 & 2,06 & 2,98 & 3,10 & $0,92 \mathrm{~ns}$ & $24,30 * *$ & $28,17 * *$ \\
\hline
\end{tabular}

** = significativo em nível de $1 \%$ pelo teste "F". ns = não significativo
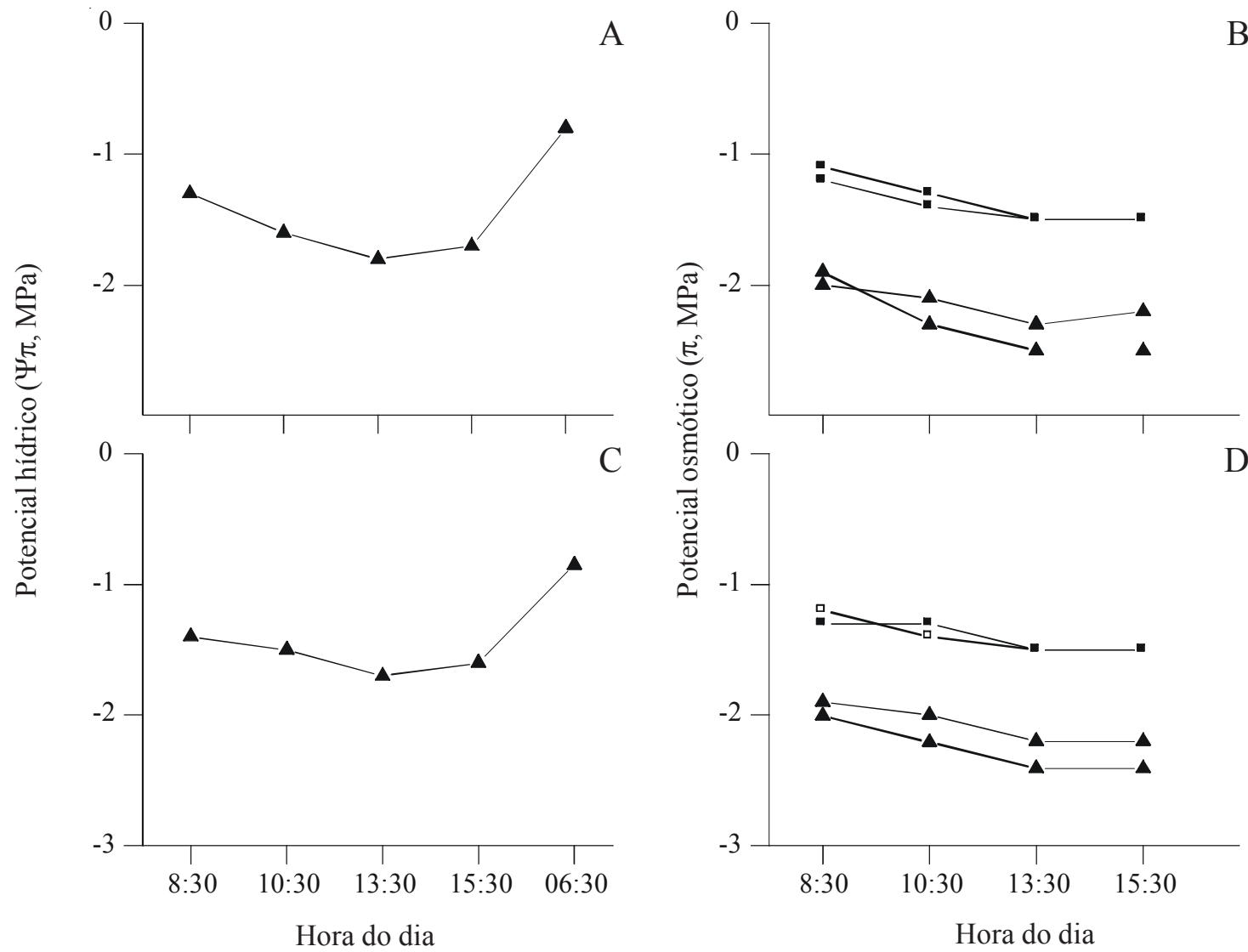

Figura 3. Potenciais hídrico $(\psi)$ e osmótico $(\psi \pi)$ nas cultivares RRIM 600 (A e B) e Fx 3864 (C e D) de Hevea brasiliensis (Willd. ex A. Juss.) Müll. Arg., nos períodos chuvoso (dez/01 - $\square$ - e fev/02 - --) e seco (jul/02 - $\triangle$ - e ago/02 - $\Delta$-). Cada ponto representa a média de quinze determinações.

Figure 3. Water $(\psi)$ and osmotic $(\psi \pi)$ potentials of cultivars RRIM 600 (A and B) and Fx 3864 (C and D) of the H. brasiliensis

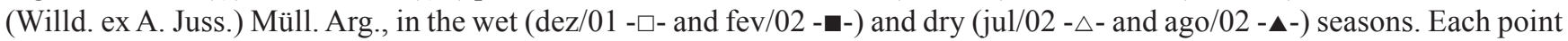
represents a mean of fifteen determinations. 
perdas. Este tipo de adaptação pode ser alcançado em genótipos com sistemas radiculares profundos (Turner 1986, Chaves 1991), como a seringueira (Mendes et al. 1992).

Curva de resposta a radiação fotossinteticamente ativa As curvas de A-RFA (figura 4) indicam comportamento similar entre as cultivares, com variações marcantes entre os períodos estudados, conforme verificado nas equações de ajuste, período chuvoso $\left(\mathrm{A}=13,37\left[1-\mathrm{e}^{-0,0018(\mathrm{x}-22,84)}\right] ; \mathrm{A}=12,45\left[1-\mathrm{e}^{-0,0017(\mathrm{x}-22,87)}\right]\right), \mathrm{e}$ no período seco $\left(A=7,43\left[1-\mathrm{e}^{-0,002(\mathrm{x}-35,82)}\right]\right.$; $\left.\mathrm{A}=6,78\left[1-\mathrm{e}^{-0,0021(\mathrm{x}-41,39)}\right]\right)$, para RRIM 600 e Fx 3864, respectivamente. Nataraja \& Jacob (1999), trabalhando com 12 clones de seringueira, com 18 meses de idade, na Índia, sob condições de laboratório, observaram taxas fotossintéticas entre 10 e $14 \mu \mathrm{mol} \mathrm{m}^{-2} \mathrm{~s}^{-1}$, para valores de $I_{s}$ entre 500 e $1.200 \mu \mathrm{mol} \mathrm{m}^{-2} \mathrm{~s}^{-1}$, comportamento similar ao verificado no presente estudo. No período chuvoso, os valores mais altos de $\mathrm{A}, \mathrm{I}_{\mathrm{s}}, \mathrm{I}_{\mathrm{c}}$ e $\varnothing$ ocorreram provavelmente devido à maior disponibilidade de água no solo, à maior quantidade de enzimas relacionadas com a assimilação de carbono na folha e à maior capacidade de transporte eletrônico entre os fotossistemas (Nataraja \& Jacob 1999).

Durante o período seco, ocorreu decréscimo da A, de $44 \%$ e $45 \%$, da $I_{s}$ de $12 \%$ e $16 \%$, do $I_{c}$ de $70 \%$ e $81 \%$ e da $\varnothing$ de $29 \%$ e $26 \%$ para RRIM 600 e Fx 3864 , respectivamente. Em termos percentuais, esta diminuição foi semelhante para ambas as cultivares. A menor taxa da fotossíntese líquida e os menores valores

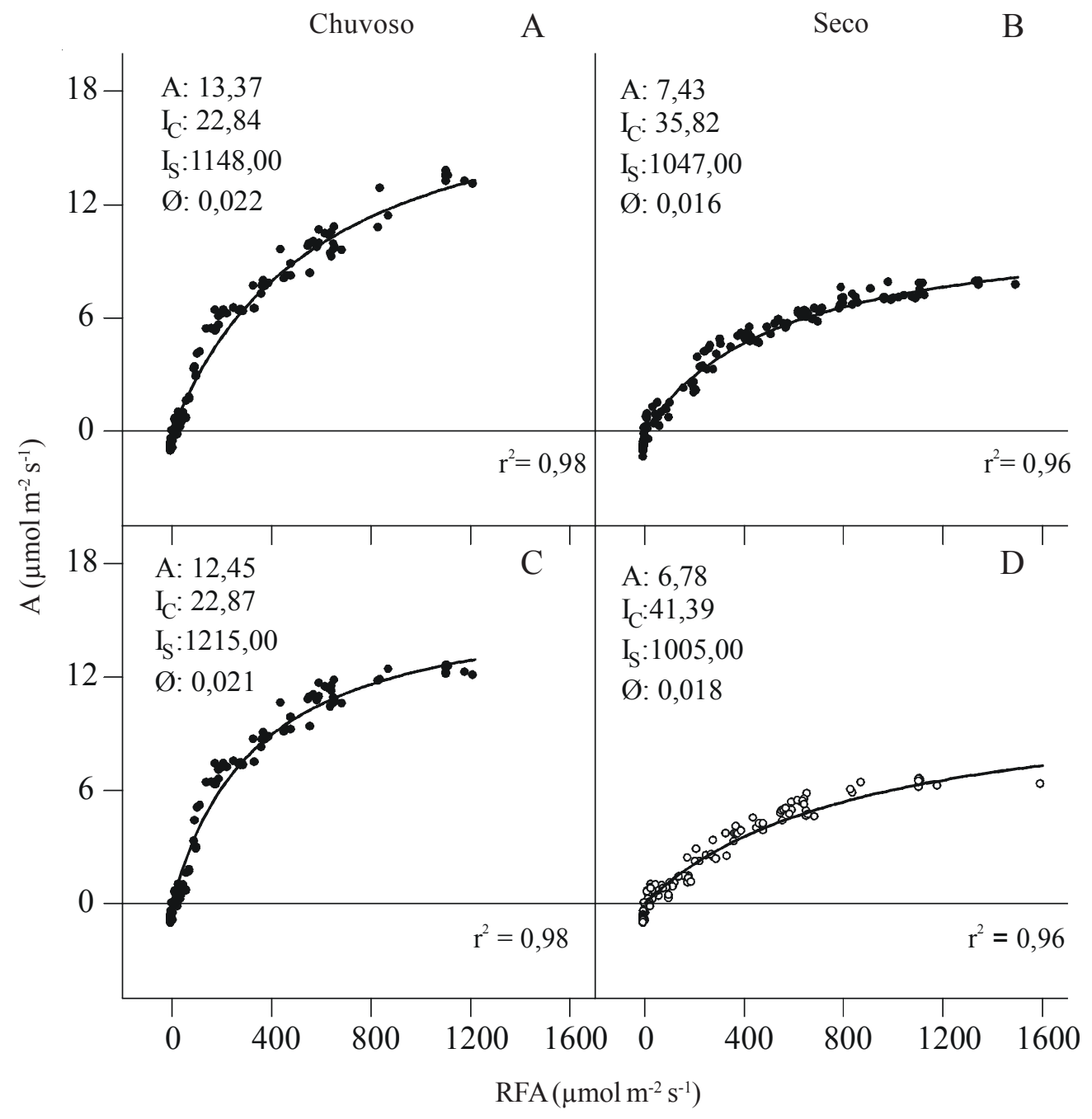

Figura 4. Taxa de fotossíntese líquida (A) em função da radiação fotossinteticamente ativa (RFA), nos períodos chuvoso (A e C) e seco (B e D) nas cultivares RRIM 600 (A e B) e Fx 3864 (C e D) de Hevea brasiliensis (Willd. ex A. Juss.) Müll. Arg.

Figure 4. Net photosynthesis rate (A) as a function photosynthetic active radiation (PAR), in the wet (A and C) and dry (B and D) seasons, of cultivars RRIM 600 (A and B) and Fx 3864 (C and D) of the H. brasiliensis (Willd. ex A. Juss.) Müll. Arg. 
de $\varnothing$ sugerem que tanto a fase bioquímica como a fotoquímica foram afetadas pelas condições desfavoráveis no período seco. A diminuição nestes valores, em parte, resulta da menor demanda por assimilados, uma vez que, nesse período o crescimento é muito baixo. Como as curvas de A-RFA foram realizadas entre $9 \mathrm{~h}$ e $10 \mathrm{~h}$, a condição hídrica foliar foi menos favorável no período seco para este horário (figura 3).

Portanto, a cultivar Fx 3864 apresentou respostas similares das trocas gasosas, $\psi$ e $\psi \pi$ foliar durante os períodos seco e chuvoso, sugerindo comportamento equivalente à RRIM 600 .

Agradecimentos - À Fapesp pelo financiamento do projeto (Processo n. 01/02217-0). A Maruceli, Alcione, Gustavo Cavalcante e Regiane Peres pelo auxílio e apoio nos experimentos de campo.

\section{Referências bibliográficas}

BATAGLIA, O.C., CARDOSO, M., IGUE, T. \& VAN RAIJ, B. 1987. Desenvolvimento da seringueira em solos do Estado de São Paulo. Pesquisa Agropecuária Brasileira 22:419-424.

BRUNINI, O. \& CARDOSO, M. 1998. Efeito do déficit hídrico do solo sobre o comportamento estomático e potencial da água em mudas de seringueira. Pesquisa Agropecuária Brasileira 33:1053-1060.

CASCARDO, J.C.M., OLIVEIRA, L.E.M. \& SOARES, A.M. 1993. Disponibilidade de água e doses de gesso agrícola nas seleções hídricas de seringueira. Revista Brasileira de Fisiologia Vegetal 5:31-34.

CAVALCANTE, J.R. \& CONFORTO, E.C. 2002. Desempenho de cinco clones jovens de seringueira na região do planalto ocidental paulista. Bragantia 61:237-245.

CHAVES, M.M. 1991. Effects of water deficits on carbon assimilation. Journal of Experimental Botany 42:1-16.

CONCEIÇÃO, H.E.O., OLIVA, M.A., LOPES, N.F. \& ROCHA NETO, O.G.R. 1985. Resistência à seca em seringueira. I Balanço hídrico e produção primária em seis clones submetidos a déficit. Pesquisa Agropecuária Brasileira 20:1041-1050.

CONFORTO, E.C., CAVALCANTE, J.R., PESSOA, J.D.C., MORENO, R.M.B. \& MATTOSO, L.H.C. 2005. Variação sazonal das trocas gasosas, turgescência relativa do tronco e produtividade em dois cultivares de seringueira em Votuporanga, SP, Brasil. Acta Botanica Brasilica 19:733-740.

CORTEZ, J.V. \& BENESI, J.F.C. 2000. Contribuição do Estado de São Paulo para o aumento da produção de borracha natural. Secretaria de Agricultura e Abastecimento Câmara Setorial de Borracha Natural. São Paulo.
DAI, Z., EDWARD, G.E. \& KU, M.S.B. 1992. Control of photosynthesis and stomatal condutance in Ricinus communis L. (Castor bean) by leaf to air vapor pressure deficit. Plant Physiology 99: 1426-1434.

EAMUS, D., MYERS, B., DUFF, G. \& WILLIAMS, D. 1999. Ecophysiological traits of deciduous and evergreen woody species in the seasonally dry tropics. Tree Physiology 14:11-16.

EMPRESA BRASILEIRADE PESQUISAAGROPECUÁRIA - EMBRAPA. 1999. Sistema Brasileiro de Classificação de Solos. Brasília, Embrapa Produção da Informação.

GONÇALVES, P.S. 1998. Recomendação de clones de seringueira para o estado de São Paulo. I Ciclo de Palestras Sobre Heveicultura Paulista. Campinas.

INSTITUTO AGRONÔMICO DE CAMPINAS. 2002. Importância da Cultura de Seringueira. http://www.iac.br/ $\sim$ rrim600/importcult.htm (acesso em 15/11/2002).

KRAMER, P.J. \& BOYER, J.S. 1995. Water relations of plants and soils. Academic Press, San Diego.

LONG, S.P. \& HALLGREN, J.E. 1985. Measurement of $\mathrm{CO}_{2}$ assimilation by plants in the field and in the laboratory. In Techniques in Bioproductivity and Photosynthesis. $2^{\mathrm{a}}$ ed. (J. Coombs, D.O. Hall, S.P. Long \& J.M. Scurlock, eds.). Pergamon Press, Oxford, p.62-94.

MACHADO, C.E. \& LAGÔA, A.M.M.A. 1994. Trocas gasosas e condutância estomática em três espécies de gramíneas. Bragantia 53:141-149.

MENDES, M.E., VILLAGRA, M.M., SOUZA, M.D., BACCHI, O.O.S. \& REICHARDT, K. 1992. Relações hídricas em seringal do município de Piracicaba, SP. Scientia Agricola 49:103-109.

MICROCAL ORIGIN. 1995. Origin Version 4.0. Microcal Software, Inc: Northampton, MA, USA. Número de Série 6025888 .

NATARAJA, K.N. \& JACOB, J. 1999. Clonal differences in photosynthesis in Hevea brasiliensis Müll. Arg. Photosynthetica 36:89-98.

NEUMANN, H.H. \& THURTELL, G.W.A. 1972. A peltier cooled thermocouple dew point hygrometer for in situ measurement of water potential. In Psychrometry in water relations research (R.W. Brow \& B.B. Van Haveren, eds.). Utah State University, Logan, p.103-112.

PRADO, C.H.B.A. \& MORAES, J.A.P.V. 1997. Photosynthetic capacity and specific leaf mass in twenty woody species of Cerrado vegetation under field conditions. Photosynthetica 33:103-112.

PRADO, C.H.B.A., PASSOS, E.E.M. \& MORAES, J.A.P.V. 2001. Photosynthesis and water relations of six tall genotypes of Cocos nucifera in wet and dry seasons. South African Journal of Botany 67:169-176.

ROCHA NETO, O.G., CANO, M.A.O. \& TIEBAULT, J.T.L. 1983. Eficiência do uso da água em plântulas de seringueira submetidas a déficit hídrico. Pesquisa Agropecuária Brasileira 18:336-339. 
SCHOLANDER, P.F., HAMMEL, H.T., BRADSTREET, E.D. \& HEMMINGSEN, E.A. 1965. Sap pressure in vascular plants. Negative hydrostatic pressure can be measured in plants. Science 148:339-346.
TURNER, N.C. 1986. Crop water deficits: a decade of progress. Advances in Agronomy 39:1-51.

ZAR, J.H. 1999. Biostatistical analysis. Prentice Hall, Upper Saddle River. 